\title{
Une femme et la guerre à la fin du Moyen Âge. Le "Livre des faits d'armes et de chevalerie" de Christine de Pizan, D. Demartini, C. Le Ninan, A. Paupert et M. Szkilnik (éds)
}

\section{Paola Cifarelli}

\section{OpenEdition}

\section{Journals}

Édition électronique

URL : https://journals.openedition.org/studifrancesi/9901

DOI : 10.4000/studifrancesi.9901

ISSN : 2421-5856

Éditeur

Rosenberg \& Sellier

Édition imprimée

Date de publication : 1 août 2017

Pagination : 339-341

ISSN : 0039-2944

\section{Référence électronique}

Paola Cifarelli, « Une femme et la guerre à la fin du Moyen Âge. Le "Livre des faits d'armes et de chevalerie" de Christine de Pizan, D. Demartini, C. Le Ninan, A. Paupert et M. Szkilnik (éds)», Studi Francesi [En ligne], 182 (LXI | II) | 2017, mis en ligne le 01 août 2017, consulté le 21 septembre 2021. URL : http://journals.openedition.org/studifrancesi/9901; DOI : https://doi.org/10.4000/studifrancesi. 9901

Ce document a été généré automatiquement le 21 septembre 2021.

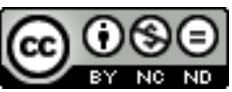

Studi Francesi è distribuita con Licenza Creative Commons Attribuzione - Non commerciale - Non opere derivate 4.0 Internazionale. 


\title{
Une femme et la guerre à la fin du Moyen Âge. Le "Livre des faits d'armes et de chevalerie" de Christine de Pizan, D. Demartini, C. Le Ninan, A. Paupert et M. Szkilnik (éds)
}

\author{
Paola Cifarelli
}

\section{RÉFÉRENCE}

Une femme et la guerre à la fin du Moyen Âge. Le "Livre des faits d'armes et de chevalerie" de Christine de Pizan. Études réunies par Dominique DEMARTINI, Claire LE NINAN, Anne PAUPERT et Michelle sZKILNIK, Paris, Champion, 2016, «Études Christiniennes» 13, 231 pp.

1 On ne peut que féliciter les collaborateurs de ce volume collectif pour avoir consacré des études ponctuelles au Livre des faits d'armes et de chevalerie, composé par Christine de Pizan autour de 1410 et seul de ses ouvrages qui attend encore une édition critique; en effet, comme les éditeurs le soulignent dans l'Introduction (pp. 7-27), «la richesse de cet ouvrage, son caractère insolite et pourtant pertinent, son étonnante destinée» (p. 27) méritaient d'être mis en lumière, afin que l'importance de la réflexion politique développée dans le traité, l'originalité de son style et l'efficacité de la technique de compilation des sources puissent ressortir pleinement. Un autre grand mérite du présent volume est la cohérence qui lie les différentes contributions, constituant autant de chapitres d'une véritable petite monographie sur le texte christinien. Les articles sont organisés en quatre sections harmoniquement structurées et consacrées respectivement à La tradition manuscrite (pp. 29-70), aux questions concernant Histoire et 
Politique (pp. 73-116), aux techniques de Compilation et réécriture (pp. 119-162) et à La Réception (pp. 165-203). En outre, la Bibliographie qui clôt le volume est un instrument de travail précieux pour ceux qui voudront se pencher sur ce texte et permet de mesurer l'intérêt que cet ouvrage a suscité, par delà une méconnaissance apparente.

2 La première section s'ouvre sur les remarques de Gabriella PARUSSA (Genèse et fortune d'un texte: la tradition textuelle du "Livre des fais d'armes et de chevalerie" de Christine de Pizan, pp. 31-47) à propos de la tradition manuscrite, à la fois touffue et complexe; les recherches menées sur les 22 témoins permettent d'éclaircir quelques-unes des nombreuses questions laissées ouvertes par les études pionnières de Charity Cannon Willard et des savants qui ont abordé la question à une époque plus récente: la répartition des témoins dans les groupes $\mathrm{A}$ et $\mathrm{B}$ est donc établie de manière fiable, tandis que des jalons importants sont posés pour résoudre les problèmes liés à la composition du traité et à sa transmission; en particulier, la collation partielle effectuée par G.P. semblerait remettre en question l'antériorité du ms Bruxelles, KBR 10476 par rapport au ms Paris, Bnf, fr. 603; en outre, le problème des corrections et modifications auctoriales ou par des scribes-remanieurs dans plusieurs témoins amène à envisager une édition numérique et interactive, pouvant rendre compte des stratifications du texte. Par contre, l'étude de Christine RENo (Les deux exemplaires originaux subsistants du "Livre des faits d'armes et de chevalerie": différences principales, pp. 51-53) reprend en partie les arguments exposés dans l'Album Christine de Pizan pour prouver que le manuscrit bruxellois conserverait la version la plus ancienne du traité, ce qui confirme que la reconstruction des dynamiques de composition pose des difficultés considérables. Le contexte manuscrit des Faits d'armes dans trois des quatre manuscrits-recueils parvenus jusqu'à nous (Paris, BnF fr. 603 et Bruxelles, KBR 9009-900: groupe A; et Bordeaux, BM 815: groupe B) font l'objet de la contribution de Karen FRESCO (Trois recueils manuscrits contenant les "Faits d'armes": Paris, BnF, fr. 603, Bruxelles, KBR 9009-9011 et Bordeaux, Bibl. Mun. 815, pp. 55-87); l'A. souligne l'affinité thématique et iconographique des Faits d'armes et de la Mutacion réunis dans le ms de Paris, tandis que le codex bruxellois, qui conserve aussi l'Arbre de Batailles d'Honorat Bovet et les Manières de Bataille de 1306, témoignerait surtout de la renommée durable de Christine de Pizan en tant qu'auteur traitant des questions concernant la chevalerie. Enfin, le manuscrit de Bordeaux associe les Faits d'armes à des ouvrages traitant eux aussi de la légitimité du pouvoir royal.

3 Les contributions de la deuxième section abordent des questions liées au droit de la guerre, au droit d'armes et aux mécanismes du commandement au sein d'un modèle de service militaire professionnalisé. Thierry LASSABATIÈRE ( La fonction de connétable et le commandement militaire dans le "Livre des faits d'armes et de chevalerie" de Christine de Pizan, pp. 73-87) souligne la nécessité d'une utilisation prudente du traité comme source complémentaire susceptible d'apporter un éclairage sur la place réelle du connétable dans l'armée: miroir du prince visant l'éducation et l'édification, il répond à une pratique culturelle privilégiant la compilation et l'imitation des anciens, plus qu'à une volonté de décrire exactement la réalité de son temps. Par contre, la conjugaison de sources antiques (Végèce, Valère Maxime et Frontin) et modernes (l'Arbre de Batailles d'Honoré Bonet, fondé à son tour sur le traité latin du juriste bolonais Giovanni da Legnano) permet à Christine de bâtir une réflexion approfondie sur la discipline militaire, le comportement des membres de l'armée, le rôle fondamental du prince dans la réglementation de l'usage de la force armée au sein d'une conception de la guerre juste (Loïc CAZAUX, Pour un droit de la guerre? La discipline militaire et les rapports 
entre combattants et non-combattants dans le "Livre des faits d'armes et de chevalerie" de Christine de Pizan, pp. 89-102). L'analyse des trois derniers chapitres de la partie IV, consacrés au droit d'armes, permet enfin à Inès VILLELA-PETIT de montrer la dette de Christine envers le Tractatus de insigniis et armis de Bartolo da Sassoferrato à travers le philtre de l'Arbre de Batailles, ainsi que d'analyser les enjeux des choix que Christine fit pour les armes imaginaires de son père (La dame à la biche: Christine de Pizan et le droit d'armes, pp. 103-115).

4 C'est la qualité littéraire de l'ouvrage christinien qui est au cœur des contributions de Claire LE NINAN ( SSy fais hardiement et ne de doubtes»: Christine de Pizan entre clergie et chevalerie dans le "Livre des faits d'armes et de chevalerie", pp. 119-132), de Bernard RiBÉmont (Le "Livre des faits d'armes et de chevalerie": droit et didactique, pp. 133-147) et d'Hélène BIU ("Et la gist la maistrie»: de l'“Arbre des Batailles" au "Livre des faits d'armes et de chevalerie", pp. 149-162); en effet, les traits caractéristiques du talent d'écrivain de Christine apparaissent, selon C.L.N., dans l'association étroite entre sagesse et chevalerie symbolisée par le personnage de Minerve, dans la valorisation de l'acte d'écriture, ainsi que dans la technique du dialogue, destiné à rendre efficaces les enseignements théoriques et pratiques et à faciliter un processus d'identification des lecteurs avec le narrateur. Le «rapport de binarité stratifiée» (p. 133) que Christine instaure avec ses lecteurs et avec sa matière, utilisée en contrepoint pour faire en sorte que l'art de chevalerie dialogue avec la morale, le droit et la loi, est considéré par B.R. comme l'élément permettant de passer du traité au manuel d'enseignement, animé et vivifié par le talent littéraire de l'auteur. En particulier, dans les parties fondées sur l'Arbre des Batailles l'étude des techniques mises en œuvre par Christine pour sélectionner la matière, la recomposer dans un raisonnement linéaire et l'inscrire dans la fiction littéraire dialogique permet à H.B. de mettre en évidence à quel point le travail de réécriture sert la clarté de l'exposition, l'efficacité, mais aussi une esthétique personnelle caractérisée par un souci d'élégance stylistique et de richesse lexicale.

$5 \quad$ Les Faits d'armes et de chevalerie régénèrent donc la matière issue de sources différentes à travers une réécriture dont la qualité littéraire a sans doute favorisé le succès durable de ce texte. Dans la dernière section, Michelle szKILNIK (Le "Jouvencel" ou le "Roman des faits d'armes et de chevalerie", pp. 165-178) prouve que l'influence du traité christinien sur le Jouvencel de Jean de Bueil s'étend bien au delà de l'emprunt ponctuel repéré depuis longtemps par la critique; les convergences se manifestent tant au niveau idéologique que sur le plan formel, à tel point qu'on peut légitimement parler de «mise en fiction» (p. 166). La fortune anglaise du Livre aux approches de la défaite dans la guerre de Cent Ans, et particulièrement le projet éditorial conçu par John Talbot dans le célèbre «Shrewsbury book», ont donné lieu à une série d'interprétations qu'Andrew TAYLOR évoque pour montrer que la réception de cet ouvrage s'insère dans un contexte où l'héritage culturel français est l'objet d'un débat serré au sein de la société anglaise ( Dame Christine» et la chevalerie savante en Angleterre, pp. 179-190). Enfin, Liliane DULAC et Earl Jeffrey RICHARDS (Le "Livre des faits d'armes et de chevalerie": une critique féminine cachée de la chevalerie?, pp. 190-203) reviennent sur la question ouverte de la suppression, dans les manuscrits du groupe $\mathrm{B}$, de toute référence à Christine pour proposer que la «masculinisation» serait la conséquence «d'une répartition des rôles de l'homme et de la femme à la cour bourguignonne, sous une forme nostalgique et traditionnelle [...] mais non pas misogyne» (p. 192) après la chute de Constantinople: la disparition, dans le prologue de la partie I, de l'évocation de Minerve symbolisant 
l'union entre sapientia et fortitudo se justifierait dans le contexte de la promotion de la croisade en 1454, réalisée à travers la métaphore de Dame Eglise se plaignant et des chevaliers de la Toison d'Or la défendant. Cependant, cette hypothèse ne pourra être considérée comme vraisemblable que lorsqu'on aura vérifié que tous les onze mss du groupe B ont été confectionnés dans la seconde moitié du siècle.

6 Par la richesse des différentes contributions, qui concourent toutes à approfondir la connaissance de ce texte complexe, ce volume rendra les plus grands services non seulement aux spécialistes de la production christinienne, mais aussi à tous ceux qui s'intéressent à l'histoire des mentalités et à la littérature du moyen âge tardif. 